\title{
Remaining Useful Life and Performance Reliability Online Assessment
}

Susan Lu*

Department of System Science and Industrial Engineering, Binghamton University, New York, USA

*Corresponding author: Susan Lu, Department of System Science and Industrial Engineering, Binghamton University, New York, USA, Tel: 6077774908; E-mail: slu@binghamton.edu

Rec date: June 09, 2014; Acc date: June 11, 2014; Pub date: June 21, 2014

Copyright: @ 2014 Lu S. This is an open-access article distributed under the terms of the Creative Commons Attribution License, which permits unrestricted use, distribution, and reproduction in any medium, provided the original author and source are credited.

Remaining useful life and performance reliability are instantaneous reliability indices for a system in operation. It is key characteristics of a system or component, especially for a mission-critical system, operating under a dynamic environment. Nowadays, as system's complexity and reliability requirement continue increasing, there is a practical need for on-line real-time reliability monitoring and assessment, associated with decision making on predictive maintenance strategy. It is also significantly beneficial to the system management in using real-time reliability prediction to reveal the system's future probability of success or failure at any operation time. Traditional reliability approaches are based on off-line analysis of life test data from a particular population under predefined operation circumstances $[1,2]$. Even though these population reliability characteristics are of great interest to manufacturers and users, there is less information to an individual system's health state in service, which is more concerned about the online reliability characteristics of the system in operation [3] and its degradation.

Traditional population-based models reflect statistical average characteristics of the system reliability under defined operation conditions $[1,2]$. Nonetheless, reliability is significantly influenced by many external and internal factors, such as conditions of operation and maintenance, inherited quality/reliability characteristics of each individual system/component, and the interface with other systems/ components, and even performance of human operators. These factors are usually highly dynamical and vary from one system to another. Under such circumstances, applying the population-based static model to each individual will result in inaccurate reliability information and lead to wrong or unfavorable maintenance/replacement decisions, which will result in increased downtime, economic loss, and even catastrophic events. According to the Electrical Power Research Institute (EPRI), just in electrical power systems, one third of total cost spent on maintenance, around $\$ 60$ billion per year, was wasted [4] due to inappropriate reliability estimation and maintenance policy. The timely and accurate evaluation of the system's reliability could provide valuable information for proactive and cost-effective maintenance planning, repair/replacement scheduling, logistic planning, etc. Furthermore, the conditional reliability at a given time is also estimated from the system remaining useful life (RUL) assessed, which plays a critical function on system remanufacturibility in remanufacturing engineering management. Remanufacturing engineering further impacts on green environment and energy sustainability development.

System remaining useful life and the performance reliability assessment are based on the system degradation with usage and aging. The degradation is a stochastic process. The performance reliability is assessed by the probability of the degradation that is reaching to or exceeding over a predefined threshold of a soft-failure defined in the degradation. Performance measures/variables are variables that are highly correlated with a system's underlying degradation, which are monotonically changing over the time. A system's performance can generally be evaluated by one or more performance measures (physical variables). Behavior changes of performance variables over time could provide valuable information about the system's degradation and health states. Over the past few decades, there has been vigorous research on system deterioration monitoring and applications. Conceptually, the system performance can be measured directly from degradation or indirectly from correlated variables that are the indices of it. For those cases where the direct measure is not available from performance degradation, then an indirect measure from correlated variable are usually adopted.

Focus and interest in this research direction are the development of theories, methodologies, and a computer system prototype for system performance reliability monitoring, forecasting, and predictive condition-based maintenance planning. This computer monitoring system, a type of online 'reliability odometer', is capable to indicate the conditional reliability at any given time instance. It is a real-time system in the domain of multivariate performance measures (multiple inputs) and multiple failure modes (multiple outputs) (MIMO). The system performance measures will serve as the model inputs. The outputs of the model will be the predicted performance reliability, the probabilities of each potential failure mode, a suggested maintenance plan, and the assessment of the remaining useful life. The remaining useful life of the system will also indicate the time of system to shut down for key components to remanufacture. Because of high nonlinearity of the stochastic degradation process, the online assessing usually adopts a state-space model with nonlinear stochastic filtering. The system maintenance plan will be based on predicted conditions in terms of failure probabilities and the associated costs of downtime, failure, maintenance, labor, etc. This methodology with state space model and nonlinear filtering will work for general nonlinear, timevariant, non-Gaussian dynamic system. Particle sequence Monte Carlo method is developed to provide online recursive estimation for both model parameter and the system state estimation for reliability dynamics. Performance reliability prediction and RUL estimate are intended for system prognostic and health management (PHM), and the decision on the time for components to remanufacture in remanufacturing management. The proposed approach will overcome the disadvantages of fixed-time period maintenance and available maintenance strategies commonly used in industry. It will advance the traditional reliability and maintainability methods from a static model to a dynamical environment, from off-line estimation to an on-line prediction, and from population attention to each individual consideration. The approach could significantly enhance system performance and minimize life cycle costs. 
Citation: Lu S (2014) Remaining Useful Life and Performance Reliability Online Assessment. Ind Eng Manage 3: e126. doi: 10.4172/2169-0316.1000e126

Page 2 of 2

There is a significant research need in development of a new integrated dynamic model that will support an online implementation for real-time performance reliability monitoring and forecasting, predictive condition-based maintenance plan, and system remaining useful life assessment. To implement the functions for 'system reliability odometer, the following research issues should be addressed:

- Methodology and algorithm development with special focus on time variant parameter (TVP) models, which enable to forecast multiple performance measures in real-time.

- Methodology and algorithm development for identifying key performance measures and their analytical or knowledge-based relationship with system's multiple competing failures.

- Methodology development of stochastic optimization of predictive condition-based maintenance policy (PCBM) based on real time performance reliability monitoring and forecasting, and RUL, which is applicable for MIMO systems. Cost model and decisionmaking model for maintenance needs to be tackled as well.

- Methodology development of signal sensing network and advanced DAQ system, and of signal preprocessing and feature attraction for performance variable online monitoring.
- Technology development of wireless signal transmission and data storage for online assessment database.

With the rapid development of stochastic nonlinear filtering technology and the computing speed improvement, an effective computer system for complex system online reliability and remaining useful life assessment will be soon shown in industrial and military applications.

\section{References}

1. Kolarik WJ (1995) Creating Quality: Concepts, Systems, Strategies, and Tools, McGraw-Hill, Inc, New York.

2. Meeker WQ (1998) Statistical Methods for Reliability Data, John Wiley \& Sons, Inc, New York.

3. Chinnam RB (1999) On-Line Reliability Estimation of Individual Components, Using Degradation Signals. IEEE Transactions on Reliability 48:.403-412.

4. Becker KC, Byington CS, Forbes NA, Nickerson GW (1998) Prediction and Preventing Machine Failures, The industrial Physicist: 20-23. 